\title{
Oncological outcome after free jejunal flap reconstruction for carcinoma of the hypopharynx
}

\author{
Jimmy Yu Wai Chan • Velda Ling Yu Chow • \\ Richie Chiu Lung Chan · Gregory Ian Siu Kee Lau
}

Received: 21 July 2011 / Accepted: 3 November 2011 / Published online: 18 November 2011

(C) The Author(s) 2011. This article is published with open access at Springerlink.com

\begin{abstract}
It has been a common practice among the oncologist to reduce the dosage of adjuvant radiotherapy for patients after free jejunal flap reconstruction. The current aims to study potential risk of radiation to the visceral flap and the subsequent oncological outcome. Between 1996 and 2010, consecutive patients with carcinoma of the hypopharynx requiring laryngectomy, circumferential pharyngectomy and post-operative irradiation were recruited. Ninety-six patients were recruited. TNM tumor staging at presentation was: stage II (40.6\%), stage III (34.4\%) and stage IV (25.0\%). Median follow-up period after surgery was 68 months. After tumor ablation, reconstruction was performed using free jejunal flap (60.4\%), pectoralis major myocutaneous (PM) flap (31.3\%) and free anterolateral thigh (ALT) flap (8.3\%). All patients underwent adjuvant radiotherapy within 6.4 weeks after surgery. The mean total dose of radiation given to those receiving cutaneous and jejunal flap reconstruction was $62.2 \mathrm{~Gy}$ and $54.8 \mathrm{~Gy}$, respectively. There was no secondary ischaemia or necrosis of the flaps after radiotherapy. The 5-year actuarial loco-regional tumor control for the cutaneous flap and jejunal flap group was: stage II (61 vs. $69 \%, p=0.9)$, stage III ( 36 vs. $46 \%, p=0.2$ ) and stage IV (32 vs. $14 \%$, $p=0.04)$, respectively. Reduction of radiation dosage in free jejunal group adversely affects the oncological control in stage IV hypopharyngeal carcinoma. In such circumstances, tubed cutaneous flaps are the preferred
\end{abstract}

J. Y. W. Chan $(\bowtie) \cdot$ V. L. Y. Chow · R. C. L. Chan · G. I. S. K. Lau Division of Head and Neck Surgery, Department of Surgery, Queen Mary Hospital, University of Hong Kong Li Ka Shing Faculty of Medicine, 102 Pokfulam Road, Hong Kong SAR, China e-mail: chanjyw@gmail.com reconstructive option, so that full-dose radiotherapy can be given.

Keywords Free jejunal flap - Carcinoma of hypopharynx · Radiation tolerance

\section{Introduction}

Defects created after circumferential pharyngectomy poses special challenges to reconstructive surgeons. The segment of upper digestive tract between the oropharynx and esophagus is lost, and the conduit has to be restored for the passage of food during future swallowing.

Various options of reconstruction are available, with variable outcomes and complications as published in the literature. According to our recently published experience, reconstruction using the free jejunal flap resulted in the lowest early post-operative pharyngocutaneous fistula rate as well as significantly lower risk of long-term anastomotic stricture and higher chance of resumption of normal diet after surgery, when compared with reconstruction using cutaneous flaps such as the tubed pectoralis major (PM) flap or the free anterolateral thigh (ALT) flap [1]. Post-operative adjuvant radiotherapy was indicated in the majority of the patients. However, radiation doses greater than $45 \mathrm{~Gy}$ are generally considered to be above the tolerance of the small bowel, whereas the effective dose range for post-operative radiotherapy for squamous cell carcinoma of the head and neck region is between 57 and $63 \mathrm{~Gy}$ [2]. This retrospective study aims to compare the outcomes of patients having jejunal flap and those with cutaneous flap reconstruction with regard to post-radiotherapy complications, loco-regional tumor control as well as survival. 

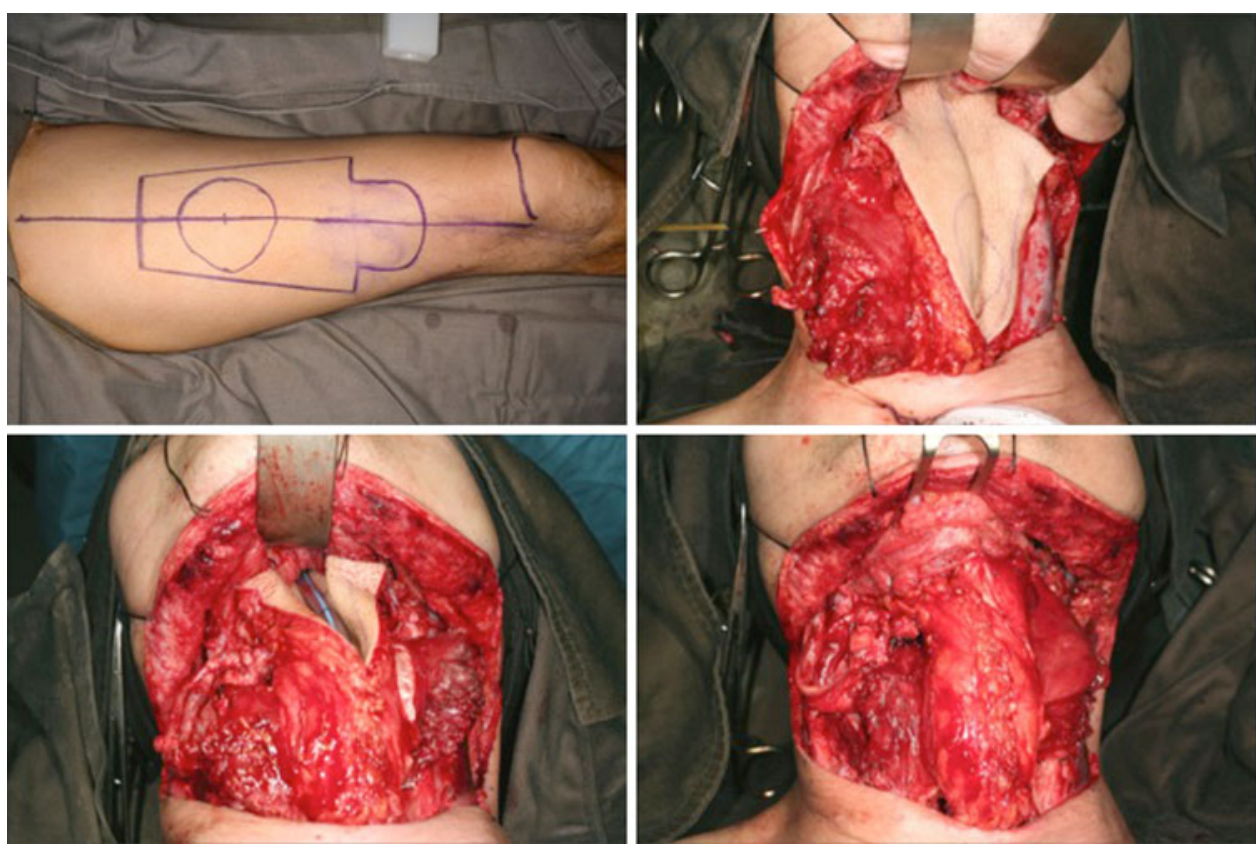

Fig. 1 Above, left A trapezoid skin island of the anterolateral thigh flap was designed, which was subsequently folded upon itself to form a tube for anastomosis between the oropharynx above and the cervical esophagus below. The semi-circular extension of the skin island is used to resurface the posterior wall of the oropharynx and the nasopharynx, which was also resected in this patient. Above, right The anastomosis

with the esophagus was completed first in order to immobilize the flap for microvascular anastomosis. Below, left Further suturing of the flap, which was progressively fashioned into a tube. Nasogastric tube was inserted for post-operative feeding. Below, right The inset of the flap is completed after anastomosing to the oropharynx

\section{Patients and methods}

From January 1996 and December 2010, at Division of Head and Neck Surgery, Department of Surgery, The University of Hong Kong at the Queen Mary Hospital, we had treated 96 patients with advanced tumors at the hypopharynx. Those with history of previous radiotherapy to the neck were excluded.

All patients have pre-operative work-up for tumor staging, anesthetic assessment and nutritional build-up if necessary. Subsequently, they underwent total laryngectomy and circumferential pharyngectomy for tumor extirpation. Cervical lymph node dissection was carried out if there was lymphatic metastasis. Intra-operative frozen section examination of the resection margins was performed to ensure microscopic clearance of disease.

The resultant circumferential pharyngeal defect between the oropharynx above and the esophagus below was reconstructed with either the cutaneous flaps [pectoralis major (PM) flap and free anterolateral thigh (ALT) flap] or the free jejunal flap. The choice of the reconstructive methods depended on surgeons' preference as well as patients' factors such as medical co-morbidities, donor site availability and the presence of suitable recipient blood vessels in the neck to support microvascular free tissue transfer. The surgical techniques of the respective reconstructive options

were described in detail in previous publications [3-16]. Essentially, for cutaneous flap reconstruction, the rectangular skin island of the PM or ALT flap was designed and fashioned into a tube to replace the upper digestive tract that had been resected with the tumor (Fig. 1). For visceral flap reconstruction, the segment of the jejunum supplied by the second arcade of vessels was harvested and used (Fig. 2). All patients received nasogastric tube feeding for nutritional support during early post-operative period. Gastrograffin swallow study was performed 1 week after surgery to ensure satisfactory wound healing before commencing oral feeding. Regular endoscopic examination was performed to check the perfusion status of the flaps. Those patients with significant delay of post-operative adjuvant chemoradiotherapy secondary to anastomotic leakage or necrotic flap requiring multiple surgeries were excluded. All patients were referred to clinical oncologists for post-operative adjuvant chemoradiotherapy.

External beam radiotherapy was commenced as soon as possible after all the surgical wounds were healed. Radiation was delivered by Cobalt $60,4 \mathrm{MV}$, or $6 \mathrm{MV}$ linear accelerator, which was given once per day with daily fraction size of $2 \mathrm{~Gy}, 5$ days per week. The spinal cord was shielded after 4,500 cGy and the flap for the pharyngeal reconstruction was included in the high-dose field of radiation. 
Fig. 2 Above, left The segment of the jejunum supplied by the second arcade of blood vessels was chosen. The vascular anatomy of the bowel was clearly visualized with light shining from behind. Above, right Meticulous dissection of the vascular arcade and the pedicle of the flap from the mesentery were then performed. Below, left The required length of the jejunum was harvested with the supplying vascular arcade, which was ready to be transferred to the neck for inset. Below, right Upon completion of flap inset. Note that the jejunum should be position in an isoperistaltic direction to facilitate future swallowing. Furthermore, redundancy of the flap should be avoided to prevent dysphagia
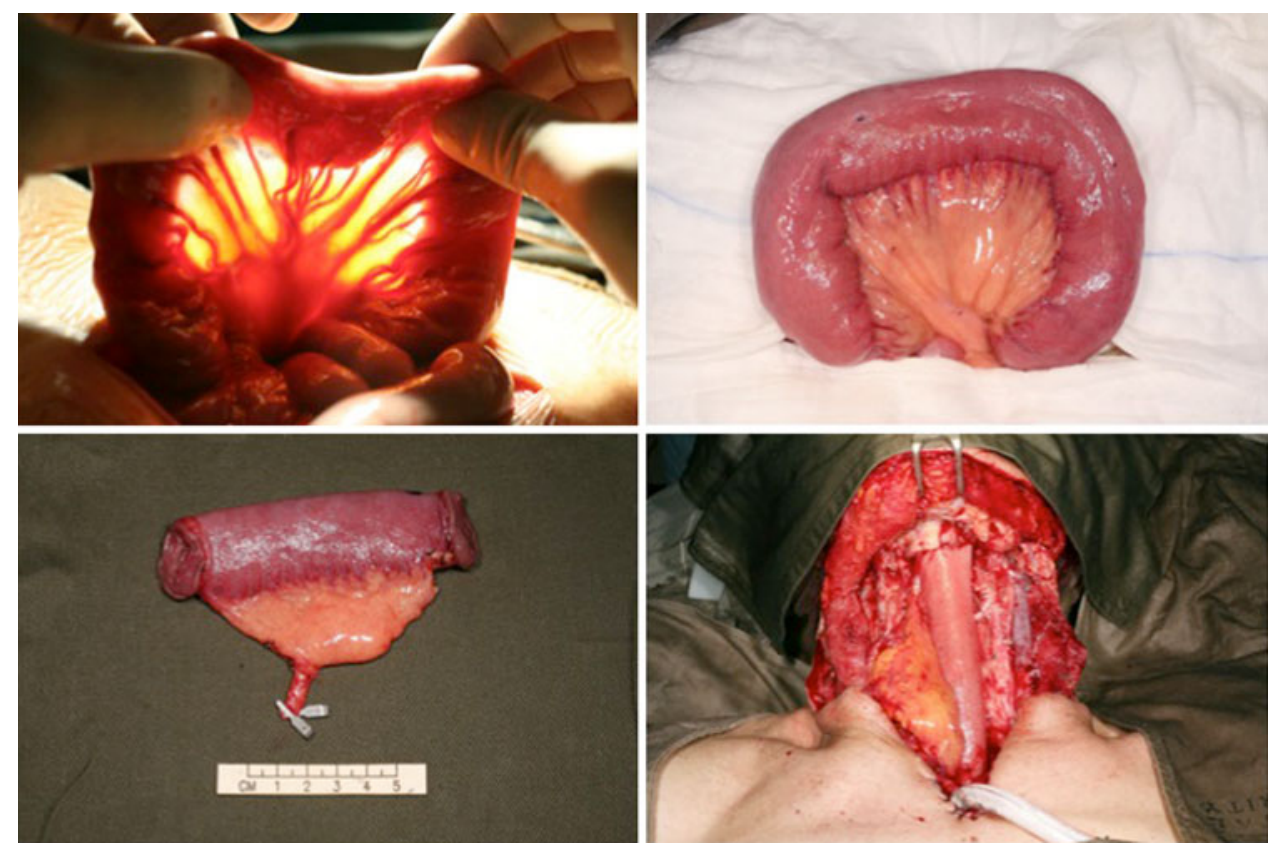

Patients were followed-up regularly with clinical, endoscopic and magnetic resonance imaging (MRI) examinations. The data forming the basis of the current study were collected prospectively. The demographic data of these patients, the dose of adjuvant radiotherapy given for patients with different types of reconstruction, the incidence of complications such as anastomotic leakage and bowel perforation were analyzed. A stage-to-stage comparison of the loco-regional tumor control was performed between different methods of reconstruction. Statistical package for social science (SPSS v. 17) was used to the analysis of the data. A $p$ value $<0.05$ was regarded as statistically significant.

\section{Results}

We have treated 96 patients during the study period, among which, 83 were males and 13 were females. The age at presentation ranged from 32 to 86 years, with a median age of 68 years. The median follow-up period was 68 months (range 5-174 months). Majority $(n=72,75.0 \%)$ of them have the primary tumor arising from the hypopharynx. The rest of the patients have recurrent tumors at the neopharynx after previous laryngectomy for carcinoma of the larynx $(n=24,25.0 \%)$. The TNM staging on presentation was stage II in $39(40.6 \%)$ patients, stage III in $33(34.4 \%)$ patients and stage IV in $24(25.0 \%)$ patients.

Circumferential resection of the pharynx was performed for all patients. In the majority $(83.3 \%)$ of the patients, we were able to achieve microscopically clear resection margins. In the rest of the patients, the surgical margins were either close $(n=12,12.5 \%)$ or involved by dysplasia or carcinoma in situ $(n=4,4.2 \%)$, as confirmed by the histological examination of the paraffin section of the resected specimen. Selective neck dissection (level II-IV) was performed in 72 sides of the neck, and microscopic lymph node metastasis was found in 14 (19.4\%) occasions. Radical neck dissection or modified radical neck dissection was performed in 58 sides of the neck. None of the patients had macroscopic residual tumor after surgery. There was no significant difference in the distribution of $\mathrm{R} 0$ or $\mathrm{R} 1$ resection among patients with different stages of disease.

After tumor ablation, 58 (60.4\%) patients received free jejunal flap reconstruction of the circumferential pharyngectomy defect. The mean length of the jejunal flap harvested was $12.6 \mathrm{~cm}$. The remaining patients had the defects reconstructed using cutaneous flaps, either the tubed PM flap $(n=30,31.3 \%)$ or tubed free ALT flap $(n=8,8.3 \%)$. There was no hospital mortality. The mean time between operation and the start of radiotherapy was 6.4 weeks. All patients completed the course of post-operative radiotherapy, and $67.7 \%$ of them had concomitant chemotherapy given as well. The mean total dose of external radiotherapy given was 62.2 Gy for patients with cutaneous flap reconstruction and 54.8 Gy for those with free jejunal flap reconstruction.

Majority $(n=84,87.5 \%)$ of the patients were able to resume oral feeding during early post-operative period before the commencement of radiotherapy. Among them, 12 patients had temporary impairment of swallowing due to the development of mucositis during radiotherapy, and nasogastric was reinserted for nutritional support. All patients eventually restored eating per orally within 
Table 1 The 5-year actuarial loco-regional tumour control rate

\begin{tabular}{llll}
\hline & $\begin{array}{l}\text { Cutaneous flap } \\
\text { reconstruction (\%) }\end{array}$ & $\begin{array}{l}\text { Free jejunal flap } \\
\text { reconstruction (\%) }\end{array}$ & $p$ value \\
\hline Stage II & 61 & 69 & 0.9 \\
Stage III & 36 & 46 & 0.2 \\
Stage IV & 32 & 14 & 0.04 \\
\hline
\end{tabular}

2 months upon completion of radiotherapy and none of them require prolonged nasogastric tube or gastrostomy feeding. There was no radiation induced salivary leakage or flap necrosis. On long-term follow-up, symptomatic stricture formation requiring regular endoscopic dilatation occurred in 8 (26.7\%) patients with tube PM flap, 1 $(12.5 \%)$ patient with tubed ALT flap and $1(1.7 \%)$ patients with free jejunum flap reconstruction.

Table 1 showed the stage-to-stage comparison of the 5year actuarial loco-regional tumor control between the two groups of patients receiving cutaneous flap and jejunal flap reconstruction. There was no significant difference in locoregional tumor control between the two groups for stage II (61 vs. $69 \%, p=0.9$ ) and III ( 36 vs. $46 \%, p=0.2$ ) disease using different methods of reconstruction. However, the difference became statistically significant in stage IV (32 vs. $14 \%, p=0.04$ ) disease. The overall survival for our patients was 64,46 and $38 \%$ for stage II, III and IV cancer, respectively. Majority of the patients died of regional $(n=12)$ and systemic metastasis $(n=23)$, while the rest of them died from metachronous tumor (carcinoma of the lung, $n=6$; carcinoma of the oral cavity, $n=2$ ) or other unrelated causes $(n=4)$.

\section{Discussion}

The management of malignant neoplasms of the hypopharynx remains a therapeutic challenge to the head and neck surgeons despite advances in surgical techniques and chemoradiotherapy. Although organ-preserving chemoradiotherapy has gained increasing popularity over primary surgical treatment for early stage disease [17, 18], surgery remains the preferred therapeutic option for locally advanced disease. It is also the only option for salvage of failures after chemo-radiotherapy. Because of the high likelihood of microscopic residual disease despite radical surgery [19], post-operative radiotherapy is generally indicated. Evidence shows that adjuvant radiotherapy significantly improved loco-regional control as well as survival after surgery [20-24] for carcinoma at the hypopharyngeal region.

Ablative surgery inevitably creates a disruption of the upper digestive tract, and this is especially true when the location and extent of tumor necessitates circumferential removal of the oropharynx and the hypopharynx. Proper choice of reconstructive techniques is crucial so that the patients can achieve a better functional outcome. However, the voluminous literature publications of different options reflect the complex nature as well as the rapid evolution in concept and techniques. Our experience shows that free jejunal transfer is safe and reliable with a high success rate, and at the same time, the donor site morbidity is minimal at experienced hands. When compared with tubed PM flap and tubed ALT flap, the incidence of early fistula (4.6 vs. 23.9 vs. $16.5 \%$, respectively) and late stricture formation ( 2.3 vs. 27.2 vs. $12.5 \%$, respectively) is significantly lower [1]. More than $60 \%$ of the patients who received free jejunal flap reconstruction were able to resume normal, solid diet eventually. Therefore, in the selected group of suitable patients, the free jejunal flap appears to be the ideal choice for the reconstruction of circumferential pharyngeal defects.

The jejunum is a radiosensitive organ in its native site in the abdomen. Radiation injury to the gastrointestinal tract is well recognized in patients with pelvic or colorectal malignancies who received external beam irradiation up to $60 \mathrm{~Gy}$ [25-27]. Acute functional changes include diarrhea, bloating and abdominal pain, and reported late sequelae include chronic diarrhea, malabsorption with steatorrhoea, abdominal spasms, intestinal obstruction, bleeding and fistula formation [28]. Radiation enteritis is the main pathological finding, particularly involving the intermitotic cells of the small intestine [29, 30], leading to sloughing of the epithelium, submucosal fibrosis, loss of glands, villous atrophy and ulceration. It is observed that, apart from the total dose of radiation delivered, the volume of the small intestine exposed to the radiation is also important in determining the severity of the presenting symptoms of the patients.

Because of these observations, there has been generalized worry among surgeons and oncologists that similar radiation induced injury can occur to a segment of the jejunum transferred to the neck as a free flap after tumor extirpation, leading to potential risk of bowel ischaemia and necrosis. Investigation in dogs demonstrated reduced amplitude of peristalsis of the jejunal autografts as well as stricture formation after exposure to 55 Gy-equivalent dose of radiation [31]. Histological examinations of the endoscopic biopsy specimens of the jejunal flap in human subjects showed blunting and atrophy of the villi, fibrosis of the mucosal lamina propria and round-cell infiltration [32]. Similarly, in our previous study, we have demonstrated mucosal edema and extensive blunting of jejunal villi at the completion of radiotherapy [33]. Scanning electron microscopic examination revealed patchy loss of microvilli on completion and at 1 month after radiotherapy, but these features were transient. These histological sequelae after 
radiotherapy, however, do not necessarily translate into clinical consequences. Early data showed no obvious early or late complications related to the jejunal autograft after exposure to 57.6-63 Gy of radiation [34]. Other studies also echo these findings by showing no flap necrosis and satisfactory swallowing in the majority of the patients after a mean radiation dose of 59 Gy $[35,36]$. It seems like that high-dose radiation therapy delivered to the jejunal flap in the neck is safer than similar radiation given to the jejunum in its native site. As mentioned before, this may be explained by the fact that the volume of the bowel irradiated is much smaller in the neck. Moreover, serving simply as a conduit rather than the organ for absorption of digested material, the function of the jejunal flap in the neck is different from that of the gastrointestinal tract inside the abdomen.

Nevertheless, its has been a common practice among the clinical oncologists to reduce the radiation dosage for patients who had received free jejunal flap reconstruction in order to prevent radiation induced complications related to the jejunal autograft. In our current study, data from the past 15 years showed that the mean radiation dosage given to patients after visceral flap transfer was much lower than those after cutaneous flap reconstruction (54.8 vs. $62.2 \mathrm{~Gy}$ ). At this level of radiation, there was no secondary ischaemia or necrosis of the flap. Despite of the reduction of radiation dosage in the jejunal group, the difference in the 5-year actuarial loco-regional tumor control between the two groups of patients was not significant for TNM stage II and III diseases. However, for stage IV disease, patients with jejunal flap reconstruction had significantly poorer locoregional tumor control. This may be explained by the failure of the reduced dose of radiation to deal with the more widespread microscopic tumor deposits in the advanced stage of disease. Despite of the apparently better functional outcome using the jejunal flap as demonstrated in our recent study, the choice of reconstructive options should never compromise the oncological control. Further largescale study is necessary to investigate the safety of highdose external beam radiation on the visceral flap. The surgeons should also communicate with the oncologists, and if a reduced dose of radiation is to be given, then jejunal transfer should not be the first choice of reconstruction in advanced staged carcinoma of the hypopharyngeal region.

\section{Conclusions}

The loco-regional tumor control is adversely affected by the reduction of post-operative adjuvant radiation dosage in patients with stage IV carcinoma of the hypopharynx reconstructed using free jejunal flap after tumor extirpation. Therefore, tubed cutaneous flaps, which allow full-dose radiation to be given, should be the reconstructive option of choice in such circumstances.

Conflict of interest All authors confirm that there is no financial interest in the production of this work and there is no financial relationship or commercial association with the manufacturer that might pose a conflict of interest.

Open Access This article is distributed under the terms of the Creative Commons Attribution Noncommercial License which permits any noncommercial use, distribution, and reproduction in any medium, provided the original author(s) and source are credited.

\section{References}

1. Chan YW, Ng RW, Liu LH, Chung HP, Wei WI (2011) Reconstruction of circumferential pharyngeal defects after tumour resection: reference or preference. J Plast Reconstr Aesthet Surg [Epub ahead of print]

2. Peters LJ, Goepfert H, Ang KK et al (1993) Evaluation of the dose for post-operative radiation therapy of head and neck cancer: first report of a prospective randomize trial. Int $\mathbf{J}$ Radiat Oncol Biol Phys 26(1):3-11

3. Withers EH, Franklin JD, Madden JJ Jr et al (1979) Pectoralis major musculocutaneous flap: a new flap in head and neck reconstruction. Am J Surg 138:537-543

4. Jegoux F, Ferron C, Malard O et al (2007) Reconstruction of circumferential pharyngolaryngectomy using a 'horseshoe-shaped' pectoralis major myocutaneous flap. J Laryngo Otol 121:483-488

5. Fabian RL (1988) Pectoralis major myocutaneous flap reconstruction of the laryngopharynx and cervical esophagus. Laryngoscope 98:1227-1231

6. Lee KY, Lore JM Jr (1986) Two modifications of pectoralis major myocutaneous flap (PMMF). Laryngoscope 96:363-367

7. Yu P, Lewin JS, Reece GP et al (2006) Comparison of clinical and functional outcomes and hospital costs following pharyngoesophageal reconstruction with the anterolateral thigh flap versus the jejunal flap. Plast Reconstr Surg 117:968-974

8. Morshed K, Szymanski M, Golabek W (2005) Reconstruction of the hypopharynx with U-shaped pectoralis major myocutaneous flap after total pharyngo-laryngectomy. Eur Arch Otorhinolaryngol 262:259-262

9. Saussez S, Cuno A, Urbain F et al (2006) Reconstruction of circumferential oro- and hypopharyngeal defects using U-shaped pectoralis major myocutaneous flap. Otolaryngol Head Neck Surg 134:823-829

10. Sagar B, Marres AM, Hartman HM (2010) Hypopharyngeal reconstruction with an anterolateral thigh flap after laryngopharyngeal resection: results of a retrospective study on 20 patients. J Plast Reconstr Aesthet Surg 63:970-975

11. Seidenberg B, Rosenak SS, Hurwitt ES et al (1959) Immediate reconstruction of the cervical esophagus by a revascularized isolated jejunal segment. Ann Surg 149:162-171

12. Theile DR, Robinson DW, Theile DE et al (1995) Free jejunal interposition reconstruction after pharyngolaryngectomy: 201 consecutive cases. Head Neck 17:83-88

13. Disa JJ, Pusic AL, Hidalgo DA et al (2003) Microvascular reconstruction of the hypopharynx: defect classification, treatment algorithm and functional outcome based on 165 consecutive cases. Plast Reconstr Surg 111:652-660

14. Julieron M, Germain MA, Schwaab G et al (1998) Reconstruction with free jejunal autograft after circumferential pharyngolaryngectomy: eighty-three cases. Ann Otol Rhinol Laryngol 107:851-857 
15. Jones AS, Roland NJ, Husband D et al (1996) Free revascularized jejunal loop repair following total pharyngolaryngectomy for carcinoma of the hypopharynx: report of 90 patients. Br J Surg 83:1279-1283

16. Fabian RL (1984) Reconstruction of the laryngopharynx and cervical esophagus. Laryngoscope 94:1334-1350

17. Bourhis J, Overgaard J, Audry H et al (2006) Hyperfractionated or accelerated radiotherapy in head and neck cancer: a meta-analysis. Lancet 368:843-854

18. Pignon JP, Bourhis J, Domenge C, Designe L (2000) Chemotherapy added to locoregional treatment for head and neck squamous cell carcinoma: three meta-analyses of updated individual data. MACH-NC Collaborative Group. Meta-analysis of chemotherapy on head and neck cancer. Lancet 355:949-955

19. Leibel SA, Scott CB, Mohiuddin M et al (1991) The effect of localregional control on distant metastatic dissemination in carcinoma of the head and neck: results of an analysis from the RTOG head and neck database. Int J Radiat Oncol Biol Phys 21(3):549-556

20. Donald PJ, Hayes HR, Dhaliwal R (1980) Combined therapy for pyriform sinus cancer using post-operative irradiation. Otolaryngol Head Neck Surg 88:738-744

21. Vandenbrouk C, Eschwege F, De La Rochefordière A (1987) Squamous cell carcinoma of the pyriform sinus: retrospective study of 351 cases treated at the Institut Gustave-Roussy. Head Neck Surg 10:4-13

22. Vikram B, Strong EW, Shah JP, Spiro R (1984) Failure at the primary site following multimodality treatment in advanced head and neck cancer. Head Neck Surg 6:720-723

23. Vikram B, Strong EW, Shah JP, Spiro R (1984) Failure in the neck following multimodality treatment in advanced head and neck cancer. Head Neck Surg 6:724-729

24. Kramer S, Gelber R, Snow JB et al (1987) Combined radiation therapy and surgery in the management of advanced head and neck cancer: final report of study 73-03 of the Radiation Therapy Oncology Group. Head Neck Surg 10:19-30

25. Andreyev HJ (2007) Gastrointestinal problems after pelvic radiotherapy: the past, the present and the future. Clin Oncol 19:790799
26. Baglan KL, Frazier RC, Yan D, Huang RR, Martinez AA, Robertson JM (2002) The dose-volume relationship of acute small bowel toxicity from concurrent 5 -FU-based chemotherapy and radiation therapy for rectal cancer. Int J Radiat Oncol Biol Phys 52:176-183

27. Fiorino C, Alongi F, Perna L et al (2009) Dose-volume relationship for acute bowel toxicity for patients treated with pelvic nodal irradiation for prostate cancer. Int J Radiat Oncol Biol Phys 75:2935

28. Coia LR, Myerson RJ, Tepper JE (1995) Late effects of radiation therapy on the gastrointestinal tract. Int J Radiat Oncol Biol Phys 31:1213-1236

29. Sher ME, Bauer J (1990) Radiation-induced enteropathy. Am J Gastroenterol 85:121-128

30. Fenoglio-Preiser CM, Lantz PE, Listrom MB, Davis M, Rilke FO (1989) Non-neoplastic small intestine. In: Gastrointestinal pathology: an atlas and text, Raven Press, New York, pp 291-292

31. Biel MA, Maisel RH (1992) Gross morphologic and functional effects of postoperative radiation on free autografts. Laryngoscope 102(8):875-883

32. Petruzzelli GJ, Johnson JT, Myers EN et al (1991) The effect of postoperative radiation therapy on pharyngoesophageal reconstruction with free jejunal interposition. Arch Otolaryngol Head Neck Surg 117(11):1265-1268

33. Wei WI, Lam LK, Yuen PW, Kwong D, Chan KW (1998) Mucosal changes of the free jejunal graft in response to radiotherapy. Am J Surg 175:44-46

34. Cole CJ, Garden AS, Frankenthaler RA et al (1995) Postoperative radiation of free jejunal autografts in patients with advanced cancer of the head and neck. Cancer 75:2356-2360

35. Barrett WL, Gluckman JL, Aron BS (1997) Safety of radiating jejunal interposition grafts in head and neck cancer. Am J Clin Oncol 20(6):609-612

36. Handl-Zeller L, Hohenberg G, Grasl M et al (1992) Radiation of jejunal interposition in T3-T4 upper aerodigestive tumours. Eur J Cancer 28:50-53 